\title{
Performance of Polar Codes for OFDM-Based UWB Channel
}

\author{
Doaa E. El. Matary ${ }^{1}$, Esam A. Hagras ${ }^{2}$, Hala Mansour Abdel-Kader ${ }^{1}$ \\ ${ }^{1}$ Communications and Electronics Department, Faculty of Engineering, Shoubra, Benha University, Cairo, Egypt \\ ${ }^{2}$ Computer and Communications Department, Faculty of Engineering, Delta University, Mansoura, Egypt \\ Email: ^doaa_elmatary@yahoo.com
}

How to cite this paper: El. Matary, D.E., Hagras, E.A. and Abdel-Kader, H.M. (2018) Performance of Polar Codes for OFDM-Based UWB Channel. Journal of Computer and Communications, 6, 102-117. https://doi.org/10.4236/jcc.2018.63008

Received: February 21, 2018

Accepted: March 23, 2018

Published: March 26, 2018

Copyright (c) 2018 by authors and Scientific Research Publishing Inc. This work is licensed under the Creative Commons Attribution International License (CC BY 4.0).

http://creativecommons.org/licenses/by/4.0/

(c) (i) Open Access

\begin{abstract}
A multiuser Ultra Wide Band (UWB) channel suffers seriously from realistic impairments. Among this, multipath fading and interferences, such as Multiple Access Interference (MAI) and Inter Symbol Interference (ISI), that significantly degrade the system performance. In this paper, a polar coding technique, originally developed by Arikan, is suggested to enhance the BER performance of indoor UWB based Orthogonal Frequency Division Multiplexing (OFDM) communications. Moreover, Interleave Division Multiple Access (IDMA) scheme has been considered for multiuser detection depending on the turbo type Chip-By-Chip (CBC) iterative detection strategy. Three different models as Symmetric Alpha Stable $(\mathrm{S} \alpha \mathrm{S})$, Laplace model and Gaussian Mixture Model (GMM), have been introduced for approximating the interferences which are more realistic for UWB system. The performance of the proposed Polar-coded IDMA OFDM-based UWB system is investigated under UWB channel models proposed by IEEE 802.15.3a working group and compared with Low Density Parity Check (LDPC)-coded IDMA OFDM-based UWB system in terms of BER performance and complexity under the studied noise models. Simulation results show that the complexity of the proposed polar-coded system is much lower than LDPC-coded system with minor performance degradation. Furthermore, the proposed polar-coded system is robust against noise and interferences in UWB indoor environment and gains a significant performance improvement by about 5 $\mathrm{dB}$ compared with un-coded IDMA-OFDM-UWB system under the studied noise models.
\end{abstract}

\section{Keywords}

Ultra Wide Band Channel, Non-Gaussian Noise, Polar Code, Multi Carrier Approach 


\section{Introduction}

In the world of wireless communication, Ultra Wide Band (UWB) is considered as an attractive technology due to its important features as robustness towards multipath fading, high data rates, improved channel capacity, low cost, low power consumption and low-complexity devices [1]. A variety of UWB systems can be designed to use the available UWB spectrum of $7.5 \mathrm{GHz}$ [2].

The traditional design approach is known as Impulse-Radio UWB (IR-UWB) which based on a very short duration pulse in transmission. Although the impulse architectures offer relatively simple radio designs, their signals are usually impaired by fading and Inter Symbol Interference (ISI) due to multipath delay spread phenomenon which in turns leads to degradation of the overall system performance. Moreover, they provide little flexibility in spectrum management [2]. Therefore, to overcome these drawbacks, an alternative approach is known as Multicarrier UWB (MC-UWB) which based on multiple simultaneous carriers in transmission is introduced. In this type Orthogonal Frequency Division Multiplexing (OFDM) technique is employed to transmit information using orthogonal carriers [3].

Regarding to a multiuser UWB indoor environment, the performance is mainly limited by inherent noise and interferences as Multiple Access Interference (MAI) and ISI which are poorly approximated by a Gaussian distribution because of their impulsive nature. Therefore, Gaussian approximation is inefficient for UWB systems. Several alternative distributions for approximating impulsive noise in UWB systems are introduced and compared [4] [5]. These distributions have in common heavy tailed behavior of their probability density function (pdf) that considered more realistic than Gaussian distribution [5].

For multiuser detection in UWB indoor environment, a recently proposed spread spectrum multiple access scheme, Interleave Division Multiple Access (IDMA) was introduced in [6]. In this scheme, each user's chip sequence is interleaved by a user-specific distinct random interleaver which in turn reduces the MAI from other users. Furthermore, a simple Chip-By-Chip ( $\mathrm{CBC}$ ) iterative Multi User Detection (MUD) strategy is used at the receiver. These interleavers are considered the unique feature to distinguish between users for IDMA system [7]. Chip interleaving was first introduced in [7] to mitigate the burst impulsive noise disturbance. Moreover, IDMA features are: suitable for wide or narrow band transmission, low receiver cost, high power and spectral efficiency, diversity against fading and flexible rate adaptation [7] [8].

Recent papers investigate the combination of OFDM and IDMA to avoid their individual disadvantages [9] [10]. The results prove that OFDM-IDMA system inherits so many advantages over other alternatives such as robustness against MAI and ISI, and ability to handle the whole burst errors caused by impulsive noise in multipath channel. Moreover, OFDM-IDMA system has the ability to allow frequency diversity with the help of efficient Forward Error Correcting (FEC) coding technique. 
Low Density Parity Check (LDPC) codes are considered as one of the most powerful FEC techniques due to their superior error correction capability with simple iterative decoding. Hence, these codes have been utilized in most of current UWB systems [11] [12]. Furthermore, LDPC coding technique has been combined with IDMA scheme based OFDM system to overcome the multipath channel imperfections efficiently and improve the performance as described in [13]. But on the other hand, the main challenge in any Broad-band Wireless Access (BWA) system is to transmit an error sensitive application data with a good trade-off between the performance and complexity. So, in recent studies, many authors are aware of the role of different coding techniques on system performance.

Recently, Polar codes are presented as an active research which are firstly proposed by Arikan [14] using a channel polarization concept [15]. Polar codes are considered as a capacity achieving codes with straight-forward construction, very low complexity of coding and decoding as well as the non-universality characteristic [16] [17].

Therefore, Polar codes will be employed in this paper, as a channel coding, with IDMA scheme over OFDM based UWB channel corrupted by non Gaussian (impulsive) noise. Moreover, a comparative study is held between the proposed polar-coded IDMA OFDM-based UWB system and LDPC-coded system in [18] to clarify the trade-off between the system performance and complexity under the studied Gaussian and non-Gaussian noise models.

The remainder of the paper is organized as follows: Section 2 presents the UWB channel and noise models. Section 3 describes the Polar-coded OFDM-IDMA-UWB system model. The simulation results of the proposed system are introduced in Section 4 and finally Section 5 concludes the paper.

\section{UWB Channel and Noise Models}

The design of any communication system depends on studying the characteristics of the channel and noise nature. Indoor UWB environments are subjected to multipath fading and impulsive noise which have a strong effect on the system performance assessment. Hence, the accurate modeling of channel and noise is a very important issue. Such models create the facility for calculation of large and small-scale characteristics which are necessary for efficient system design [19].

\subsection{UWB Channel Model}

The standardized channel model for indoor UWB environments has been proposed by the channel modeling subcommittee of the IEEE 802.15.3a Task Group. Such model is a modified version of the Saleh-Valenzuela (S-V) model, where the Rayleigh distribution of the channel coefficient amplitude is replaced by the log-normal distribution [20]. Four different channel models (CM1, CM2, CM3, and CM4) based on the average distance between transmitter and receiver, as well as the propagation conditions were specified by the IEEE 802.15.3a sub- 
committee [20].

\subsection{Impulsive Noise Model}

The wide spread of electronic devices in indoor environments causes high level noise impulses as a form of the non-Gaussian noise [4]. That is, their pdf have heavy tailed behavior and go to zero more slowly than a Gaussian pdf. The noise in UWB channel can be modeled as [21]:

$$
n=n_{a}+n_{I}
$$

where $n$ is the total noise and can be divided into $n_{a}$ which is background (AWGN) noise with zero mean and variance $\sigma^{2}$, and $n_{I}$ which is the Impulsive noise with zero mean and variance $\sigma_{I}^{2}$. Impulsive noise $n_{I}$ can be accurately modeled by three different models, symmetric alpha stable, Laplace model, Gaussian mixture model, which will be introduced below.

\subsubsection{The Symmetric Alpha Stable Model}

According to [22], the Symmetric Alpha Stable $(S \propto S)$ distribution can be completely determined by two parameters: 1 ) a characteristic exponent $\propto \in[0,2]$, which indicates the characteristic of the tail of the $S \propto S$ distribution; 2) a divergence (or scale) parameter $\gamma \in(0, \infty)$ which is analogous to the variance of the Gaussian distribution. One difficulty of the $S \propto S$ distribution is that they have no closed-form expressions for their pdf $f_{\propto}(r)$ except for $\propto=1$ (i.e., the Cauchy distribution) and $\propto=2$ (i.e., the Gaussian distribution). The pdf of any random variable $r$ at $\alpha=1$ (Cauchy detector) can be defined as [23]:

$$
f_{\text {Cauchy }}(r)=\frac{\gamma}{\pi} \frac{1}{\gamma^{2}+(r-\mu)^{2}}
$$

where $\mu$ is the location parameter of the distribution.

\subsubsection{The Laplace Model}

The pdf of any random variable $r$ of the Laplace noise model is given by [24]:

$$
f_{\text {Laplace }}(r)=\frac{1}{2 C} \exp \left(-\frac{|r-\mu|}{C}\right),-\infty<x<\infty
$$

where $\mu$ is the location parameter (mean), and $C$ is the scale parameter ( $2 C^{2}=$ variance ) and its value is always positive. By varying the scale parameter $C$, different tail behaviors can be obtained [24].

\subsubsection{The Gaussian Mixture Model}

A simple two term mixture pdf of any random variable $r$ is given by [25] [26]:

$$
f_{G M M}(r)=(1-\epsilon) g(r)+\epsilon h(r)=\frac{(1-\epsilon)}{\sqrt{2 \pi \sigma_{g}^{2}}} \mathrm{e}^{\frac{(r-\mu)^{2}}{2 \sigma_{g}^{2}}}+\frac{\epsilon}{\sqrt{2 \pi \sigma_{h}^{2}}} \mathrm{e}^{-\frac{(r-\mu)^{2}}{2 \sigma_{h}^{2}}}
$$

where $g($.$) is the nominal Gaussian pdf with variance \sigma_{g}^{2}$ and $h($.$) is the$ heavy tailed Gaussian with variance $\sigma_{h}^{2}=\eta \sigma_{g}^{2}$, where $\eta \geq 1$ is the impulsive 
part's relative variance with respect to (w.r.to) nominal Gaussian noise variance. The parameter $\epsilon \in[0,1]$ controls the contribution of impulsive component to the whole pdf [26].

\section{Polar-Coded IDMA-OFDM-UWB System Model}

The proposed Polar-coded IDMA OFDM-based UWB system model for multi-user communication scenario is depicted in Figure 1.

\subsection{The Transmitter Structure}

For simplicity, synchronous BPSK signaling is considered over time in-variant multipath channel. For the user-k, the information data sequence $d_{k} \in\{0,1\}$ of length $D$ is encoded using polar encoder into a coded sequence $c_{k}$ of length $N$. A polar code can be completely described by three-tuples $(N, D, \mathrm{~F})$, where $N$ is the code length in bits, $D$ is the number of information bits encoded per codeword, and $\mathrm{F}$ is a subset of $N-D$ integer indices called frozen bit locations from $\{0,1, \cdots, D-1\}$ [17].

The construction of polar code depends on the choice of the subset $F$. This is corresponding to the selection of best $D$ bit-channels among $N$, in terms of the

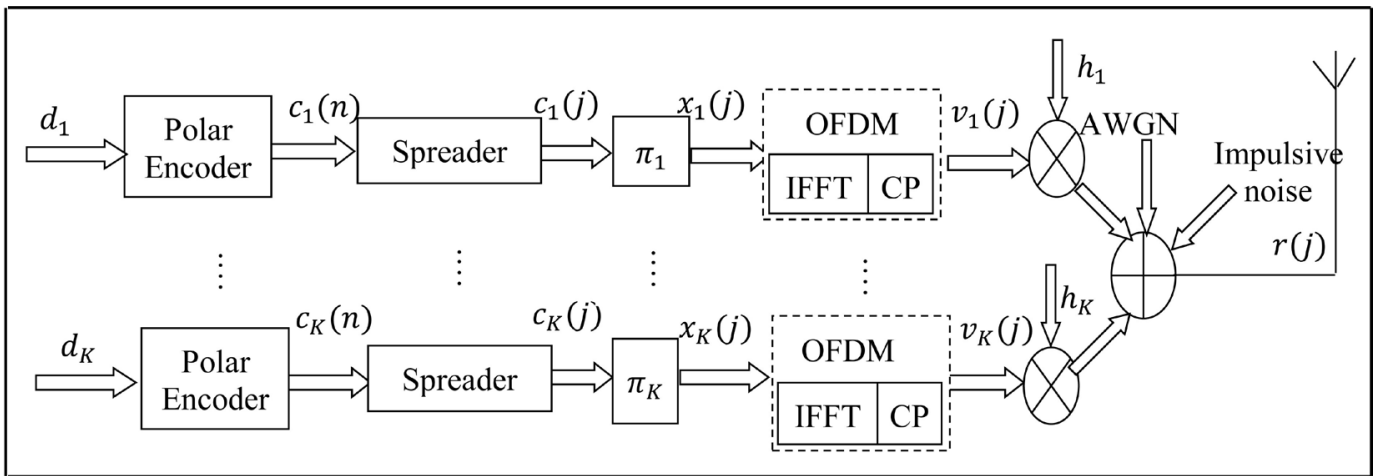

(a)

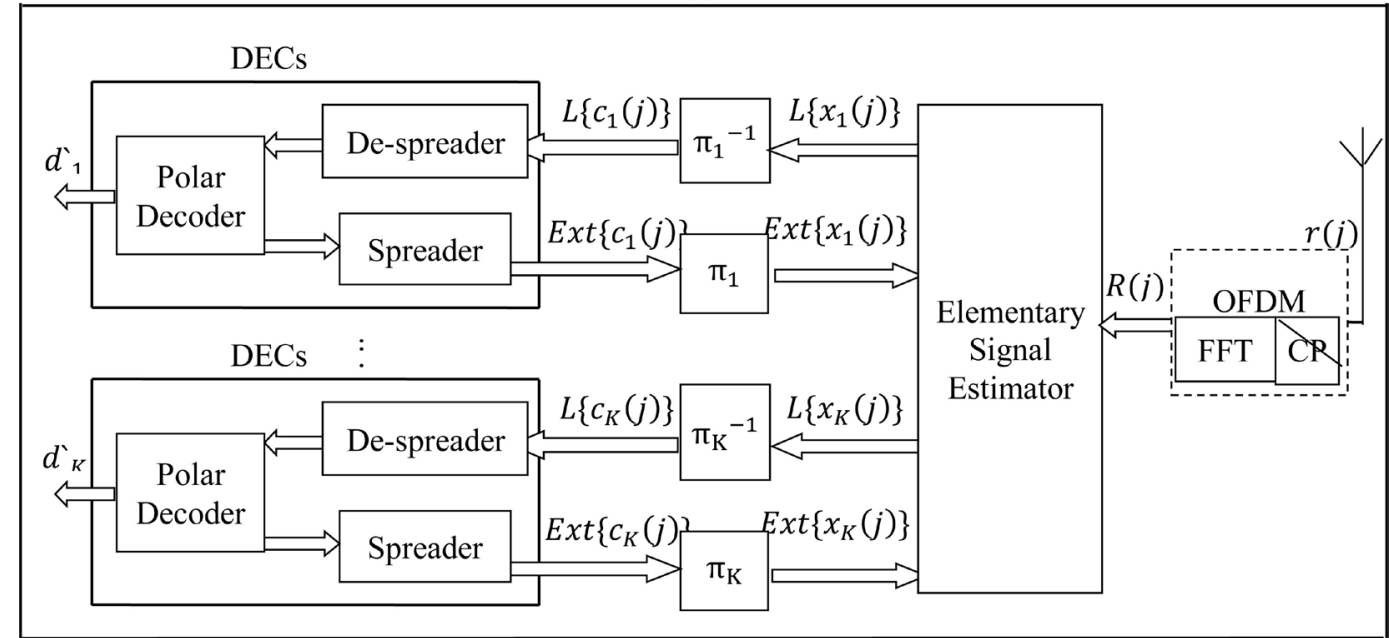

(b)

Figure 1. Polar-Coded IDMA-OFDM-UWB system model. (a) Transmitter; (b) Receiver. 
bit error rate (BER) at a given value of $\left(R E_{b} / N_{0}\right)$, where $R$ is the code rate, defined as the design-SNR [17].

The $n^{\text {th }}$ coded bit of user-k, $c_{k}(n), n=1,2, \cdots, N$ is spread using a balanced spread sequence of length $S$. The same spreading code is applied to all users $S_{k} \in\{+1,-1\}$. The obtained chip sequence is written as $\left\{c_{k}(j), j=1,2, \cdots, J\right\}$, where $J=N \times S$ is the chip length. A specific distinct chip level random interleaver $\left\{\pi_{k}, k=1,2, \cdots, K\right\}$ is employed for user separation [7] [8].

The interleaved chip sequences are mapped using BPSK onto the modulated symbols $\left\{x_{k}(j), j=1,2, \cdots, J\right\}$ which are the elements of BPSK constellation. For simplicity, the symbol mapping process is not shown in Figure 1(a). The BPSK symbols $x_{k}(j)$, considered an input to OFDM stage, are transmitted through orthogonal subcarriers by using Inverse Fast Fourier Transform (IFFT) operation, where the total number of subcarriers for OFDM transmission is denoted by $N_{c}$. In addition, a Cyclic Prefix CP of length $L_{g}$, assumed to be larger than the channel length, is inserted to prevent ISI and ICI [11].

As a result, the time-domain data sequence, including $\mathrm{CP}$, has the form:

$$
v_{k}=\frac{1}{N_{c}} \sum_{n=0}^{N_{c}-1} x_{k, n} \mathrm{e}^{i 2 \pi n t / N_{c}}, t=-L_{g}, \cdots, 0, \cdots, N_{c}-1
$$

where $x_{k, n}$ is the modulated data of the OFDM symbol of the user-k which is mapped to the $\mathrm{n}^{\text {th }}$ sub-carrier. The OFDM signal $v_{k}$ is transmitted through the multipath channel. It is assumed that the multipath channel impulses of each user, $h_{k}=\left\{h_{k}(0), h_{k}(1), \cdots, h_{k}(L-1)\right\}$, are stationary in a frame period and mutually independent, where $L$ denotes the number of resolvable paths. The output signal $r(j)$ at any time instant $j$ is a linear superposition of independently symbols for each user. The output of multipath channel is written as [11]:

$$
r(j)=\sum_{k=1}^{K} h_{k}(j) * v_{k}(j)+n(j)
$$

where $*$ denotes the convolution, $h_{k}$ the UWB channel coefficient for user-k and $n(j)=n_{a}(j)+n_{I}(j)$ the total noise.

\subsection{The Receiver Structure}

The turbo-type iterative receiver structure of the proposed system is depicted in Figure 1(b) which includes two main parts, Elementary Signal Estimator (ESE) and a bank of k single user A Posteriori Probability (APP) decoders (DECs) [13]. Successive Cancellation Decoder (SCD) of polar code, has been introduced to achieve desirable performance and complexity trade-offs [14]. At the receiver side, OFDM demodulation is carried out before iterative MUD process [10]. The received signal in frequency domain after OFDM demodulation, CP removal and FFT operation, can be expressed as

$$
R(j)=\sum_{k=1}^{K} H_{k}(j) x_{k}(j)+N(j)
$$

where $x_{k}(j)$ and $H_{k}(j)$ are respectively the data chip over the $n^{\text {th }}$ subcarrier 
and the corresponding channel tap for the user-k estimated from $H_{k}(j)=\sum_{l=0}^{L-1} h_{k}(l) \cdot \mathrm{e}^{-i 2 \pi l n / N_{c}} \cdot N(j)$, FFT of $n(j)$, are samples of noise [11]. The received signal $R(j)$ is processed iteratively by means of ESE and DECs functions which discussed as follows.

\subsubsection{The ESE Function}

The ESE performs coarse chip-by chip estimation. Re-write Equation (7), the received signal at time instant $j$ is:

$$
R(j)=\sum_{k=1}^{K} H_{k}(j) x_{k}(j)+\xi_{k}(j)
$$

where $\xi_{k}(j)=\sum_{m \neq k} H_{m}(j) x_{m}(j)+N(j)$ represents a distortion (including interference plus noise) with respect to user-k. It is assumed that the channel coefficients $H_{k}$ are known a priori at the receiver. Moreover, it is supposed that the received signal is perfectly synchronized. A CBC iterative detection based on Log Likelihood Ratio (LLR) of the received chip sequence approximated as either Gaussian or non-Gaussian noise. In case of Gaussian approximation (i.e. $n(j)=n_{a}(j)$ only) and $x_{k}(j)$ is treated as a random variable with mean $E\left(x_{k}(j)\right)$ and variance $\operatorname{Var}\left(x_{k}(j)\right)$ (initialized to 0 and 1 respectively) [10]. Then from Equation (8), we have:

$$
\begin{gathered}
E(R(j))=\sum_{k=1}^{K} H_{k}(j) E\left(x_{k}(j)\right) \\
\operatorname{Var}(R(j))=\sum_{k=1}^{K}\left|H_{k}(j)\right|^{2} \operatorname{Var}\left(x_{k}(j)\right)+\sigma^{2}
\end{gathered}
$$

where $\sigma^{2}$ is the power of the background (AWGN) occurring during the $\mathrm{j}^{\text {th }}$ chip. Using the central limit theorem [11], $\xi_{k}(j)$ in Equation (8) can be approximated by a Gaussian random variable with:

$$
\begin{gathered}
E\left(\xi_{k}(j)\right)=E(R(j))-E\left(H_{k}(j) x_{k}(j)\right) \\
\operatorname{Var}\left(\xi_{k}(j)\right)=\operatorname{Var}(R(j))-\left|H_{k}(j)\right|^{2} \operatorname{Var}\left(x_{k}(j)\right)
\end{gathered}
$$

The ESE outputs are the (LLRs) about $\left\{x_{k}(j)\right\}$ computed based on Equation (9) (using Equation (10)) as [10]:

$$
\begin{aligned}
& L\left(x_{k}(j)\right) \equiv \log \left(\frac{\operatorname{Pr}\left(x_{k}(j)=+1 \mid R(j)\right)}{\operatorname{Pr}\left(x_{k}(j)=-1 \mid R(j)\right)}\right) \\
& =\log \left(\frac{\exp \left(-\frac{\left(R(j)-E\left(\xi_{k}(j)\right)-H_{k}(j)\right)^{2}}{2 \operatorname{Var}\left(\xi_{k}(j)\right)}\right)}{\exp \left(-\frac{\left(R(j)-E\left(\xi_{k}(j)\right)+H_{k}(j)\right)^{2}}{2 \operatorname{Var}\left(\xi_{k}(j)\right)}\right)}\right) \\
& =\left(\frac{2 H_{k}(j)\left(R(j)-E\left(\xi_{k}(j)\right)\right)}{\operatorname{Var}\left(\xi_{k}(j)\right)}\right) \forall k, j
\end{aligned}
$$


In case of non-Gaussian approximation, $\xi_{k}(j)$ can be approximated using three different non-Gaussian models as, Cauchy, Laplace, and Gaussian mixture model. As Cauchy and Laplace models considered a good approximation for impulsive noise only (i.e. $\left.n(j)=n_{I}(j)\right)$ and $\xi_{k}(j)$ in Equation (8) has either Cauchy or Laplace distribution, so we have:

$$
\begin{aligned}
& E(R(j))=\sum_{k=1}^{K} H_{k}(j) E\left(x_{k}(j)\right) \\
& \operatorname{Var}(R(j)) \\
& =\sum_{k=1}^{K}\left|H_{k}(j)\right|^{2} \operatorname{Var}\left(x_{k}(j)\right)+\sigma_{I}^{2}
\end{aligned}
$$

where $\sigma_{I}^{2}$ is the power of the impulsive noise occurring during the $\mathrm{j}^{\text {th }}$ chip. While, GMM is considered a good fit for both Gaussian and non-Gaussian noise (i.e. $\left.n(j)=n_{a}(j)+n_{I}(j)\right), \xi_{k}(j)$ has GMM distribution, hence from Equation (8) we have:

$$
\begin{aligned}
& E(R(j))=\sum_{k=1}^{K} H_{k}(j) E\left(x_{k}(j)\right) \\
\operatorname{Var}(R(j)) & \\
= & \sum_{k=1}^{K}\left|H_{k}(j)\right|^{2} \operatorname{Var}\left(x_{k}(j)\right)+\sigma^{2}+\sigma_{I}^{2}
\end{aligned}
$$

For Cauchy distribution ( $\alpha=1)$, the ESE outputs are the (LLRs) about $\left\{x_{k}(j)\right\}$ as follow [22]:

$$
L\left\{x_{k}(j)\right\}=\log \left[\frac{\gamma^{2}+\left(r(j)-E\left(\xi_{k}(j)\right)+h_{k}(j)\right)^{2}}{\gamma^{2}+\left(r(j)-E\left(\xi_{k}(j)\right)-h_{k}(j)\right)^{2}}\right] \forall k, j
$$

The Cauchy detector has been used as a suboptimal detector to model the impulsive noise only with a robust performance, but it is still complex because of the need to calculate the log operation [17].

For Laplace model, the case in which the noise samples $\xi_{k}(j)$ have a Laplace distribution [4], the ESE outputs are the (LLRs) about $\left\{x_{k}(j)\right\}$ as follows:

$$
\begin{aligned}
L\left\{x_{k}(j)\right\} & =\frac{2}{C}\left(\left|\frac{r(j)}{2}-\frac{E\left(\xi_{k}(j)\right)-h_{k}(j)}{2}\right|-\left|\frac{r(j)}{2}-\frac{E\left(\xi_{k}(j)\right)+h_{k}(j)}{2}\right|\right) \\
& = \begin{cases}E\left(\xi_{k}(j)\right)-h_{k}(j) & r(j) \geq E\left(\xi_{k}(j)\right)-h_{k}(j) \\
r(j) & E\left(\xi_{k}(j)\right)+h_{k}(j)<r(j)<E\left(\xi_{k}(j)\right)-h_{k}(j) \\
E\left(\xi_{k}(j)+h_{k}(j)\right) & r(j) \leq E\left(\xi_{k}(j)\right)+h_{k}(j)\end{cases}
\end{aligned}
$$

Laplace distribution has been used as a good approximation for impulsive noise also with complexity lower than Cauchy model since it needs to calculate the sum operation only. For GMM distribution, the case in which the noise samples $\xi_{k}(j)$ have a GMM distribution, the ESE outputs are the (LLRs) about $\left\{x_{k}(j)\right\}$ as follow [26]: 


$$
L\left\{x_{k}(j)\right\}=\log \frac{\left(\frac{1-\epsilon}{\sigma} \mathrm{e}^{-\frac{\left(r(j)-E\left(\xi_{k}(j)\right)-h_{k}(j)\right)^{2}}{2 \operatorname{Var}\left(\xi_{k}(j)\right)}}+\frac{\epsilon}{\sigma_{I}} \mathrm{e}^{-\frac{\left(r(j)-E\left(\xi_{k}(j)\right)-h_{k}(j)\right)^{2}}{2 \eta \operatorname{Var}\left(\xi_{k}(j)\right)}}\right)}{\left(\frac{1-\epsilon}{\sigma} \mathrm{e}^{-\frac{\left(r(j)-E\left(\xi_{k}(j)\right)+h_{k}(j)\right)^{2}}{2 \operatorname{Var}\left(\xi_{k}(j)\right)}}+\frac{\epsilon}{\sigma_{I}} \mathrm{e}^{-\frac{\left(r(j)-E\left(\xi_{\xi}(j)\right)+n_{k}(j)\right)^{2}}{2 \eta \operatorname{Var}\left(\xi_{k}(j)\right)}}\right)}
$$

GMM is considered an excellent fit to simulation because it can adapt between AWGN and impulsive noise but with high complexity since many parameters and operations need to be estimated. After the end of ESE process, The LLR-valued chip sequence $L\left(x_{k}(j)\right)$ is then de-interleaved to produce LLRs of $c_{k}(j),\left\{L\left(c_{k}(j)\right)\right\}$ which is delivered to DECs part discussed as follows.

\subsubsection{The DEC Function}

The DECs in Figure 1(b) consists of SCD of polar code and soft in soft out CBC spread/de-spread operations. The DECs output are the extrinsic LLRs of $c_{k}(j)$, $\left\{\operatorname{Ext}\left(c_{k}(j)\right)\right\}$, which is interleaved to produce $\left\{\operatorname{Ext}\left(x_{k}(j)\right)\right\}$ and then fed back to the ESE. In the next iteration, $\left\{\operatorname{Ext}\left(x_{k}(j)\right)\right\}$ is used as a priori information to update $E\left(x_{k}(j)\right)$ and $\operatorname{Var}\left(x_{k}(j)\right)$ as [10]:

$$
\begin{gathered}
E\left(x_{k}(j)\right)=\tanh \left(\frac{\operatorname{Ext}\left(x_{k}(j)\right)}{2}\right) \\
\operatorname{Var}\left(x_{k}(j)\right)=1-E\left(x_{k}(j)\right)^{2}
\end{gathered}
$$

As discussed in Equation (10), $E\left(x_{k}(j)\right)$ and $\operatorname{Var}\left(x_{k}(j)\right)$ will be used in the ESE to update the interference mean and variance [11]. The effect of impulsive noise is regarded as interference. Due to random interleaving and de-interleaving process, the impulsive noise is spread out over the interleaving block. These interleavers disperse the resultant sequences so that the adjacent chips are approximately uncorrelated, which facilitates the simple chip-by-chip detection scheme. This iterative process is repeated a preset number of times. In the final iteration, the DECs produce a hard decision on information bit which is decoded by Polar decoder. The iteration number can be adjusted depending on the system requirements and computational power [11].

\section{Simulation Results}

In this section, the simulation results demonstrate the performance of Polar-coded IDMA OFDM-based UWB system. The discrete time channel model proposed by the IEEE 802.15.3a working group [19] is utilized, which is based on the modified S-V model. This contribution focuses on the line-of-sight (LOS) channel model 1 (CM1) which corresponds to a short-range $(0-4 \mathrm{~m})$ indoor wireless environment. The simulation results are averaged over a large number of channel realizations using the Matlab 7 program (Version 7.8.0.347). Not only AWGN is considered here but also non-Gaussian noise models like symmetric alpha stable, Laplace, and GMM. 
The simulation parameters are summarized as follows: Each user data length is 128 bits, Polar encoding is applied with rate $=1 / 2$. FFT size $=256$ and cyclic prefix $=32$ was added to each OFDM symbol block to avoid the effect of inter-chip interference. A common length-32 spreading sequence is assigned to all users, and a randomly generated chip interleaver is allocated to each user. The maximum iteration number of IDMA receiver is 3 . The simulation is performed for $(1-32)$ users. It is assumed that all users initially are synchronous with equal power allocation. The receiver is assumed to have perfect knowledge of the channel state information.

The simulation has three cases: The first case studies the BER performance of the proposed polar-coded IDMA OFDM-based UWB system for multi-users ( $k$ $=1,8,16,32)$ under Gaussian and non-Gaussian noise models. The second case presents the performance and complexity comparison between the proposed system and LDPC-coded IDMA OFDM-based UWB system introduced in [18] under the studied noise models. Finally, the last case investigates the performance comparison between un-coded-IDMA-OFDM-UWB system and the proposed system under Gaussian and non-Gaussian noise models.

Figure 2 and Figure 3 show the performance of the proposed Polar-coded IDMA-OFDM UWB system for multi users $(k=1,8,16,32)$ under the Gaussian and non-Gaussian noisy channel effect.

Figure 2(a) demonstrates the BER performance of the proposed system for multi-users under the effect of AWGN model. The results indicate the power of Polar coding technique on system performance. It can be seen that the proposed system achieves a significant improvement under Gaussian model with near single user performance for 1-16 users and degrades by about $2 \mathrm{~dB}$ for 32-users.

Figure 2(b) shows the BER performance of the proposed system for multi-users under alpha-stable noise model effect with a characteristic exponent $\propto$ $=1$ (Cauchy distribution) which confirms the heavy tailed nature of impulsive noise. The results show that the system achieves a significant improvement under this model for all users with minor degradation by about $1 \mathrm{~dB}$ compared with AWGN model.

Figure 3(a) shows the BER performance of the proposed system for multi-users under the effect of Laplacian noise model with a scale parameter $C=1$ which is commonly used to represent the tail of impulsive noise. The results indicate that the BER performance is degraded by about $1.5 \mathrm{~dB}$ compared with AWGN model.

The BER performance of the proposed system for multi-users under GMM effect is introduced in Figure 3(b). The most common values used of impulsive and control parameters for GMM are $\eta=10$ and $\epsilon=0.1$ respectively. It is observed that the performance is degraded by $2.5 \mathrm{~dB}$ with respect to AWGN model.

Furthermore, we found that by increasing the number of users to 32 , the performance of the proposed system degraded by significant amount because of increasing the MAI and inter-chip interference as shown in Figure 2 and Figure 3. 


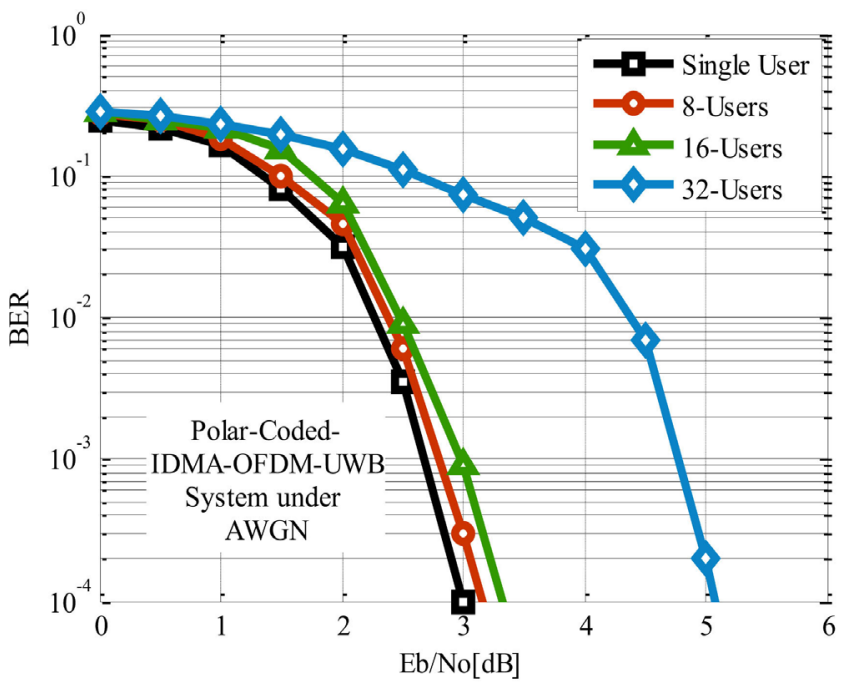

(a)

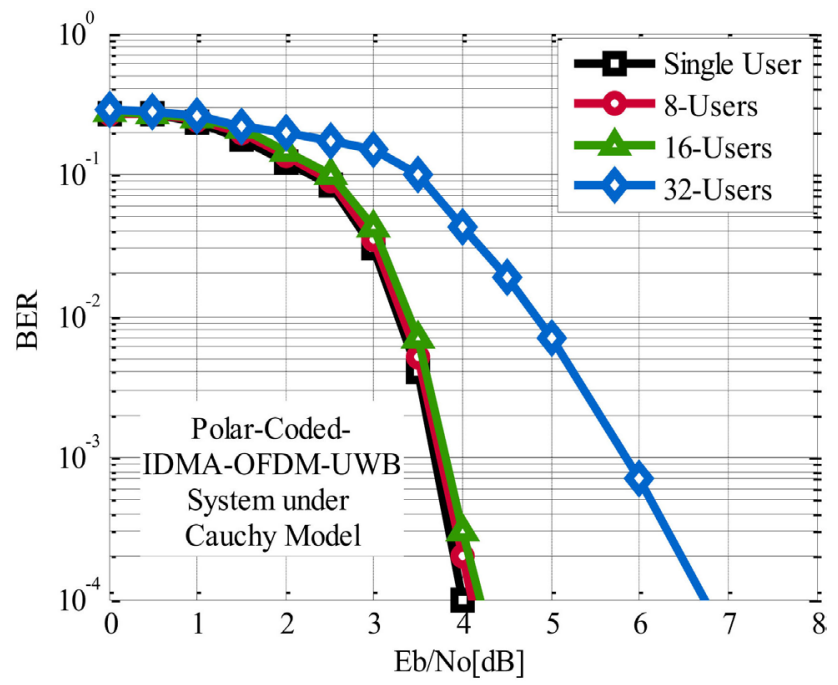

(b)

Figure 2. BER performance of Polar-coded IDMA-OFDM-UWB system for multi-users. (a) under AWGN model; (b) under Cauchy model.

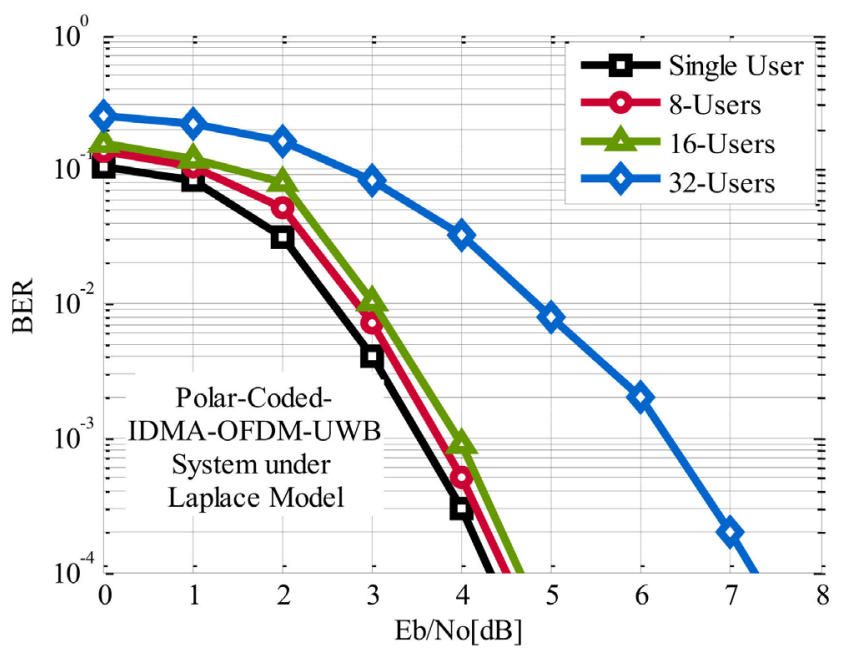

(a)

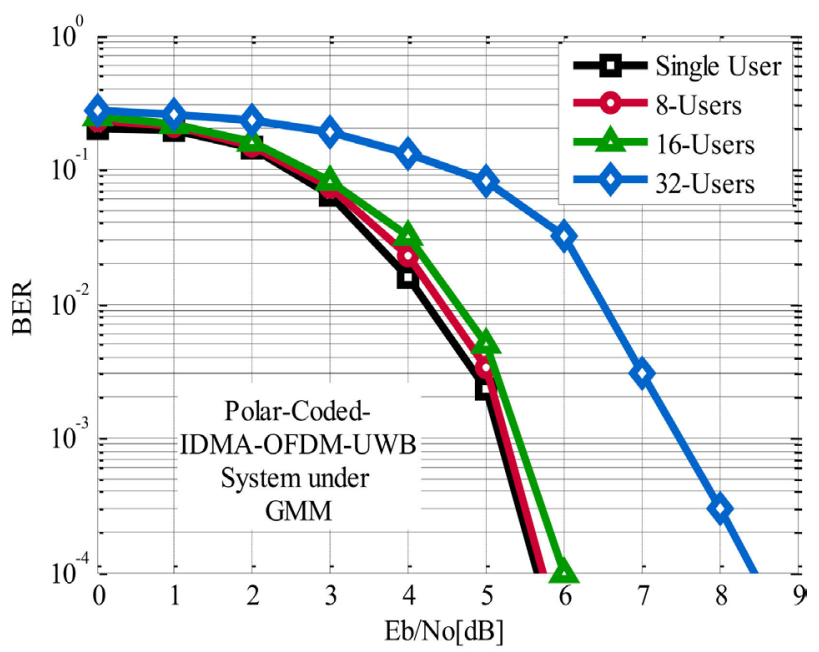

(b)

Figure 3. BER performance of Polar-coded IDMA-OFDM-UWB system for multi-users. (a) under Laplace model; (b) under GMM.

From the previous study, it can be noticed that Cauchy and Laplace models achieves a good performance compared with AWGN model than GMM. Furthermore, they are considered a good and simple approximation for impulsive noise while GMM can adapt between AWGN and impulsive noise but with high complexity since many parameters and operations needed to be estimated.

The second case of simulation shows a comparative analysis between the proposed system and LDPC-coded IDMA-OFDM-UWB system in [18] from the aspect of performance and complexity. From the performance point of view, the two systems have the common parameters as: data length of each user $N=128$ bits, code rate $=0.5$, number of users $=16$, spread length $=32$, FFT size $=256$, cyclic prefix $=32$, IDMA iteration $=3$ and for LDPC, the Sum Product Algo- 
rithm $(\mathrm{SPA})$ iteration $=7$. It can be observed from Figure 4(a) that LDPC coded system achieves a slight improvement within $(0.35-0.85) \mathrm{dB}$ for multi users compared with the proposed polar coded system under the studied noise models as shown in Table 1. But on the other hand, the polar coded system outperforms the LDPC coded system from the complexity point of view as described below.

In case of polar code, the recursive structure of channel polarization construction imposes a low complexity encoding and decoding algorithms. As in [16], the complexity of encoding and decoding of polar codes are the same of $O(N \log N)$ as a function of code block-length and independent of the code rate while that of LDPC codes are $O\left(N^{2}\right)$ for encoding and $O(N \log N)$ for decoding [27]. Therefore, the total complexity of polar code $=$ $O(N \log N)+O(N \log N)=2 O(N \log N)$ and that of LDPC code $=$ $O\left(N^{2}\right)+O(N \log N)$. Furthermore, the complexity for the LDPC decoding algorithms is $7 * O(N \log N)$ since the SPA iteration number is 7 while the complexity for successive cancellation decoder of polar code $=O(N \log N)$.

Hence the overall complexity of the system (both encoder and decoder including the iteration of IDMA) for polar codes is $3 *[2 * O(N \log N)]=6 * O(N \log N)$ while for LDPC is

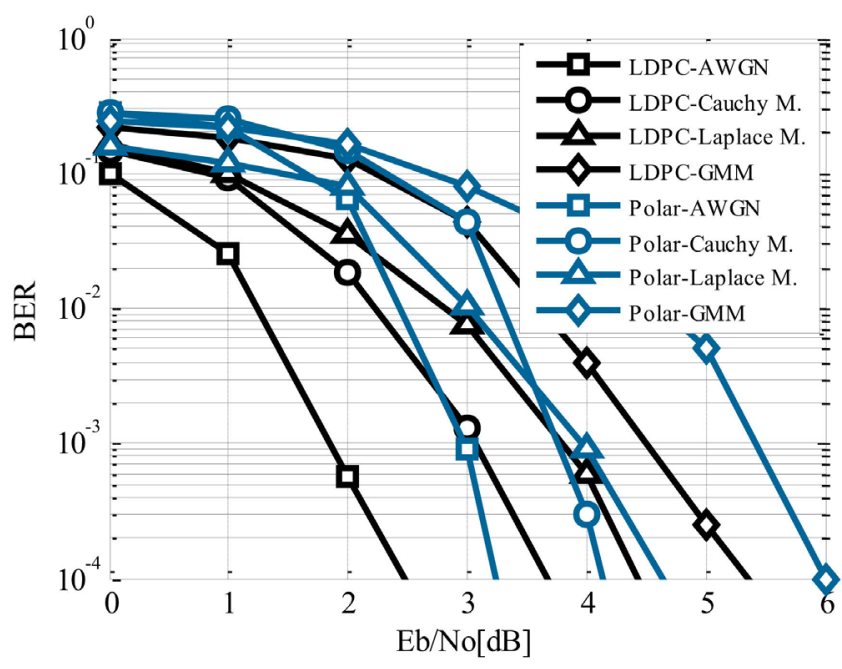

(a)

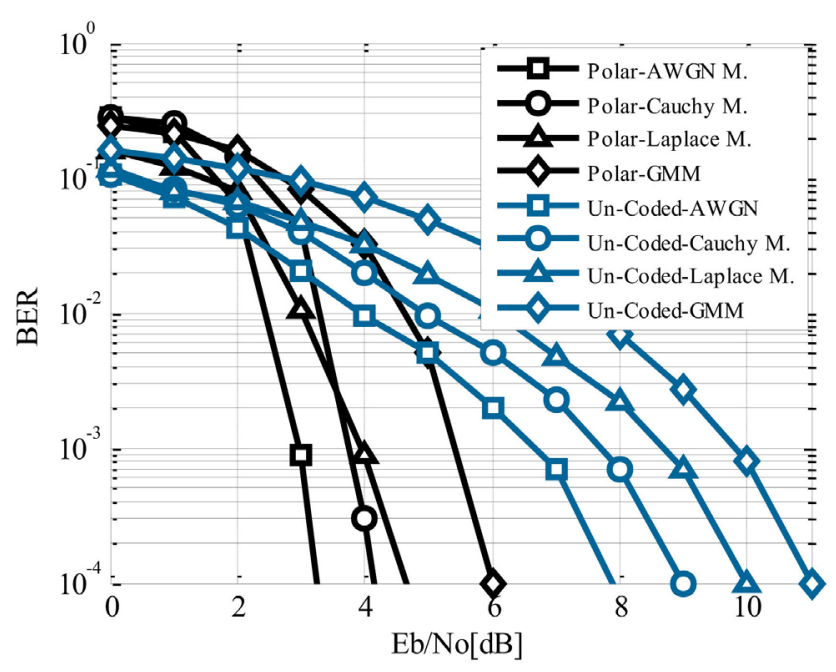

(b)

Figure 4. BER performance comparison for 16-users under the studied noise models. (a) between LDPC and Polar-coded OFDM-IDMA UWB systems; (b) between the un-coded and Polar-coded OFDM IDMA UWB systems.

Table 1. Performance Comparison between (LDPC and Polar) coded-IDMA-OFDM-UWB systems.

\begin{tabular}{cccc}
\hline System Performance & \multicolumn{2}{c}{$E_{b} / N_{0}[\mathrm{~dB}]$ at BER $=10^{-4}$} \\
\hline Noise Models & $\begin{array}{c}\text { LDPC-Coded IDMA } \\
\text { OFDM-UWB System }\end{array}$ & $\begin{array}{c}\text { Polar-Coded IDMA } \\
\text { OFDM-UWB System }\end{array}$ & $\begin{array}{c}\text { Un-Coded IDMA } \\
\text { OFDM-UWB System }\end{array}$ \\
\hline AWGN & 2.5 & 3.35 & 7.95 \\
Cauchy & 3.65 & 4.2 & 9.11 \\
Laplacian & 4.3 & 4.65 & 10 \\
GMM & 5.3 & 6 & 11 \\
\hline
\end{tabular}


$3 *\left[O\left(N^{2}\right)+7 * O(N \log N)\right]=3 * O\left(N^{2}\right)+21 * O(N \log N)$ So, despite of the performance of LDPC-coded system is better than polar-coded system, the complexity of LDPC-coded system exceeds the complexity of the polar-coded system by $3 * O\left(N^{2}\right)+15 * O(N \log N)$ which make the polar codes do better from the complexity point of view.

Finally, as shown in Figure 4(b), a comparative analysis is held between the proposed Polar-coded and un-coded-IDMA-OFDM-based UWB systems for 16-users under the studied noise models. It can be noticed that the polar-coded system achieves performance improvement within (4.6 - 5.35) dB compared with the un-coded system as demonstrated in Table 1.

Furthermore, our work outperforms the work introduced in [28] in terms of the performance and complexity. We verified that polar coded IDMA-OFDM system is robust against fading and interferences for multi-users UWB channel with better BER performance and much lower complexity compared with the system presented in [28].

\section{Conclusions}

In this paper Polar code is introduced instead of LDPC code in IDMA-OFDM based UWB system to mitigate the interferences and reduce the complexity. Moreover, a comparative analysis is held between the two systems from the aspects of performance and complexity using non-Gaussian approximation for the interferences which are more realistic for UWB system.

The comparison shows that the proposed system is robust against noise and interferences with complexity much lower than LDPC-coded system. Therefore, polar coding technique can be considered a potential candidate for the emerging $5 \mathrm{G}$ communications due to its reliability with low decoding complexity.

\section{References}

[1] Sravanthi, J. and Swathi, S. (2017) Cooperative Communication in UWB OFDM Systems. International Journal of Engineering Science Invention, 6, 60-67.

[2] Sahukar, B. (2015) Differential Code Shift Reference Impulse Radio U1tra-Wideband Transceiver: Bit Error Rate Tests and Performance Assessments. Master of Applied Science, Dalhousie University, Halifax, Nova Scotia.

[3] Montojo-Bennassar and Juan, I. (2009) Adaptive OFDM-Based UWB. Ph.D. UC San Diego Electronic Theses and Dissertations, University of California, San Diego.

[4] Beaulieu, N.C. and Young, D.J. (2009) Designing Time-Hopping Ultra-Wide Bandwidth Receivers for Multi-User Interference Environments. Proceedings of the IEEE International Conference of Communication, Beijing, 27 February 2009, 255-284.

[5] Hu, B. and Beaulieu, N.C. (2008) On Characterizing Multiple Access Interference in TH-UWB Systems with Impulsive Noise Models. Proceedings of the IEEE Radio and Wireless Symposium, Orlando, 22-24 January 2008, 879-882. https://doi.org/10.1109/RWS.2008.4463633

[6] Ping, L. (2005) Interleave Division Multiple Access and Chip By Chip Iterative Multi User Detection. IEEE Communication Magazine, 43, S19-S23. 
https://doi.org/10.1109/MCOM.2005.1452830

[7] Ping, L., Liu, L., Wu, K.Y. and Leung, W.K. (2006) Interleave Division Multiple Access. IEEE Transactions on Wireless Communications, 5, 938-947.

https://doi.org/10.1109/TWC.2006.1618943

[8] Shukla, V., Shukla, M.K. and Pande, T. (2012) Multiuser Detection Using IDMA Scheme in UWB Home Environment. International Journal of Computer Applications (0975-8887), 55, No.13. https://doi.org/10.5120/8816-2728

[9] Alagulakshmi and Arun, V. (2014) Performance Improvement of OFDM-IDMA with Modified SISO. International Journal of Research in Electronics \& Communication Technology, 2, 26-33.

[10] Yang, K. and Wang, X.D. (2006) A Multicarrier Interleave-Division UWB System. IEEE Transactions on Wireless Communications, 5, 1827-1837. https://doi.org/10.1109/TWC.2006.1673094

[11] Murugeswari, P., Janardhana, and Prabha, D. (2013) BER Performance of LDPC Code with TH-PPM over UWB Channel. International Journal of Scientific \& Engineering Research, 4, 267-271.

[12] Jayasheela, M., Rajeswari, A. and Prabha, D. (2012) Multi-User Detection Schemes for TH PPM UWB System Using LDPC Codes. International Journal of Computer Applications (0975-8887), 38, No. 11.

[13] Huang, W.-C., Lu, K.-S., Li, C.-P. and Li, H.-J. (2010) Performance Evaluation for LDPC Coded OFDM-IDMA Systems over Frequency Selective Fading Channels. IEEE Transactions on Wireless Communications, Taiwan, 16-19 May 2010, 1-5. https://doi.org/10.1109/VETECS.2010.5493931

[14] Arikan, E. (2016) On the Origin of Polar Coding. IEEE Journal on Selected Areas in Communications, 34, 209-223. https://doi.org/10.1109/JSAC.2015.2504300

[15] Tal and Vardy, A. (2013) How to Construct Polar Codes. IEEE Transactions on Information Theory, 59, 6562-6582. https://doi.org/10.1109/TIT.2013.2272694

[16] Şaşoğlu, E. (2012) Polarization and Polar Codes. Foundations and Trends in Communications and Information Theory, 8, 259-381.

[17] Vangala, H., Viterbo, E. and Hong, Y. (2015) A Comparative Study of Polar Code Constructions for the AWGN Channel. arXiv:1501.02473v1 [cs.IT]

[18] Matary, D.E.E., Hagras, E.E.A. and Abdel-Kader, H.M. (2017) Multi-User Communication Based OFDM-UWB System under Non-Gaussian Noisy Channel. American Journal of Electrical and Electronic Engineering (AJEEE), 5, 136-143. https://doi.org/10.12691/ajeee-5-4-3

[19] Molisch, A.F. (2009) Ultra-Wide-Band Propagation Channels. Proceedings of the IEEE, 97, 353-371. https://doi.org/10.1109/JPROC.2008.2008836

[20] Saleh, A.A.M. and Valenzuela, R. (1987) A statistical Model for Indoor Multipath Propagation. IEEE Journal on Selected Areas in Communication, 5, 128-137. https://doi.org/10.1109/JSAC.1987.1146527

[21] Chen, X., Qu, F.Z. and Yang, L.Q. (2011) OFDM-IDMA for Power Line Communication. 2011 IEEE International Symposium on Power Line Communications and Its Applications (ISPLC), Udine, Italy, 3-6 April 2011, 24-28.

[22] Ghannudi, H.E., Clavier, L., Azzaoui, N., Septier, F. and Rolland, P.-A. (2012) Alpha Stable Interference Modeling and Cauchy Receiver for an IR-UWB Ad Hoc Network. IEEE Transactions on Communications, 58, 1748-1757.

[23] Sayed, T. (2012) Receiver Design for Signals in Non-Gaussian Noise Applications to Symmetric Alpha-Stable and Middleton's Class-A Noise Models. Ph.D. Thesis, Car- 
leton University Ottawa, Ontario, Canada.

[24] Bao, Y.P., Zhu, Y.D., Zhu, X.M. and Chu, Y.Z. (2015) A Spectrum Sensing Method Based on Fractional Lower Order Moments in Weakly Correlated Laplace Noise. 2015 Asia-Pacific Signal and Information Processing Association Annual Summit and Conference (APSIPA), Hong Kong, China, 16-19 December 2015, 892-896. https://doi.org/10.1109/APSIPA.2015.7415399

[25] Guney, N., Delic, H. and Koca, M. (2006) Robust Detection of Ultra-Wideband Signals in Non-Gaussian Noise. IEEE Transaction on Microwave Theory and Techniques, 54, 1724-1730. https://doi.org/10.1109/TMTT.2006.872038

[26] Ayyar, A., Lentmaier, M., Giridhar, K. and Fettweis, G. (2009) Robust Initial LLRs for Iterative Decoders in Presence of Non-Gaussian Noise. 2009 IEEE International Symposium on Information Theory, Seoul, 28 June-3 July 2009, 904-908. https://doi.org/10.1109/ISIT.2009.5205626

[27] Hesami, P. (2009) Channel Polarization and Polar Codes, Capacity Achieving. University of Notre Dame, Notre Dame.

[28] Koike-Akino, T. and Wang, Y. (2017) Bit-Interleaved Polar Coded OFDM for Low Latency M2M Wireless Communications. 2017 IEEE International Conference on Communications (ICC), Paris, 21-25 May 2017, 1-7. 


\section{Nomenclature}

APP: A Posteriori Probability

AWGN: Additive White Gaussian Noise

BER: Bit Error Rate

BWA: Broad-band Wireless Access

CBC: Chip-By-Chip

CM: Channel Model

CP: Cyclic Prefix

DECs: Decoders

ESE: Elementary Signal Estimator

FEC: Forward Error Correcting

FFT: Fast Fourier Transform

GMM: Gaussian Mixture Model

ICI: Inter Carrier Interference

IDMA: Interleave Division Multiple Access

IFFT: Inverse Fast Fourier Transform

IR: Impulse Radio

ISI: Inter Symbol Interference

LDPC: Low Density Parity Check

LLR: Log Likelihood Ratio

LOS: Line of Sight

MAI: Multiple Access Interference

MC: Multicarrier

MUD: Multi User Detection

OFDM: Orthogonal Frequency Division Multiplexing

pdf: probability density function

SCD: Successive Cancellation Decoder

SNR: Signal to Noise Ratio

SPA: Sum Product Alghorithm

S-V: Saleh-Valenzuela

SaS: Symmetric Alpha Stable

UWB: Ultra Wide Band

5G: fifth Generation. 